\title{
MONITORAMENTO DA POLIMERIZAÇÃO DO PIRROL VIA ESPECTROFOTOMETRIA DE INFRAVERMELHO PRÓXIMO
}

\author{
L. M. SANTOS ${ }^{1}$, M. J. AMARAL ${ }^{1}$, C. Z. BRUSAMARELLO ${ }^{2}$, M. FORTUNY $^{1}$, A. F. SANTOS ${ }^{1}$, C. $^{2}$ \\ SAYER ${ }^{2}$, P. H. H. ARAÚJO² \\ ${ }^{1}$ Universidade Tiradentes, Programa e Pós-graduação em Engenharia de Processos, Núcleo de \\ Estudos em Sistemas Coloidais \\ ${ }^{2}$ Universidade Federal de Santa Catarina, Departamento de Engenharia Química e Engenharia de \\ Alimentos, Laboratório de Controle de Processos \\ E-mail para contato: alexfsantos@ hotmail.com
}

\begin{abstract}
RESUMO - O polipirrol é um dos mais importantes polímeros condutores por ser facilmente dopado sem a utilização de compostos altamente tóxicos e por ser termodinamicamente estável. Apresenta em sua estrutura ligações duplas conjugadas, o que lhe proporciona elevada afinidade com elétrons e baixo potencial de ionização. Neste trabalho, o espectrofotômetro de infravermelho próximo (NIR) foi proposto para predizer em linha as conversões da polimerização oxidativa do pirrol. As curvas espectrais foram associadas aos valores de conversão através da regressão linear baseada na técnica dos mínimos quadrados parciais (PLS, ou Partial Least Squares), que consiste numa técnica de regressão multivariável particular capaz de manipular entradas correlacionadas a dados limitados. Apesar da grande opacidade do meio, a técnica NIR mostrou-se adequada no acompanhamento da conversão, mesmo diante de perturbações da concentração de agente oxidante no meio.
\end{abstract}

\section{INTRODUÇÃO}

Os polímeros condutores tem despertado um amplo interesse por combinarem as propriedades químicas e mecânicas dos polímeros com as propriedades eletrônicas dos semicondutores e dos metais. São representados em uma classe de "metais sintéticos" e na sua estrutura molecular contêm ligações duplas conjugadas, que exibem propriedades como alta afinidade eletrônica, baixo potencial de ionização e podem ser facilmente reduzidos ou oxidados (SYED e DINESON, 1991; VERNITSKAYA e EFIMOV, 1997; GOSPODINOVA e TERLEMEZYAN, 1998; PRON e RONNOU, 2002; CHEN et al., 2010).

Na família dos polímeros condutores o polipirrol é um dos mais importantes por ser facilmente dopado sem a utilização de compostos altamente tóxicos e por ser termodinamicamente estável. Além disso, vem sendo utilizado para diversos tipos de aplicações, como em aparelhos eletrônicos, baterias, dispositivos de computação óptica, dispositivos 


\section{9 a 22 de outubro de 2014 \\ Florianópolis/SC}

eletrônicos, sensores e em muitas outras tecnologias avançadas (EOFINGER et al., 1998; DIAS et al., 2006; SKOTHEIM et al., 2007).

A polimerização química oxidativa é utilizada para a produção do pirrol através do acoplamento entre cátions radicais. Na etapa de iniciação, o pirrol é oxidado e convertido em um cátion-radical, ocorrendo o acoplamento e a desprotonação de dois cátions radicais que produzem o dímero de pirrol, que, por sua vez, é novamente oxidado pelo agente oxidante. Ao entrar na etapa de propagação ocorre a reoxidação, o acoplamento e a desprotonação dos dímeros sucessivamente de maneira a formar oligômeros (SKOTHEIM et al., 2007). A etapa de terminação desse sistema ainda necessita de mais estudos, mas presume-se que ocorre o ataque nucleofílico da cadeia polimérica, no qual, foi constatado por DIAS et al. (2006), através do infravermelho com transformada de Fourier (FTIR), ocorrendo uma forte absorção no comprimento de onda na região de $1700 \mathrm{~cm}^{-1}$, sugerindo o ataque nucleofílico da água e do ânion hidroxílico durante a polimerização.

Neste sentido, é essencial o uso de ferramentas de monitoramento de processos para fins de obtenção de informações sobre a evolução das propriedades do polipirrol. Em função de sua versatilidade e rapidez na predição de propriedades de correntes de processo, a técnica de espectrofotometria NIR (near infrared) tem sido utilizada com sucesso no acompanhamento de uma série de propriedades de sistemas poliméricos em tempo real (SANTOS et al., 2005; FONSECA et al., 2009). Através de procedimentos específicos de tratamento de dados espectrais e de calibração do instrumento, é possível correlacionar de maneira eficiente as propriedades de interesse com os espectros NIR, permitindo assim a inferência e o controle do processo.

Tendo em vista a escassez de estudos neste tema, bem como a falta de sensores em linha para o acompanhamento das propriedades do polipirrol, o objetivo deste trabalho é empregar um espectrofotômetro de infravermelho próximo para o monitoramento em tempo real do processo de polimerização do pirrol no acompanhamento da conversão, mesmo diante de perturbações da concentração de agente oxidante no meio.

\section{METODOLOGIA}

Nos ensaios experimentais o monômero usado foi o pirrol com $98 \%$ de grau de pureza da marca Sigma-Aldrich. O agente oxidante empregado foi o cloreto de ferro hexahidratado com o grau de pureza de 97\% da marca Sigma-Aldrich, nas concentrações de 0,1/0,25/0,5 mol, e sob a temperatura de $20^{\circ} \mathrm{C}$, com o intuito de estudar uma nova metodologia para o monitoramento do sistema. A água miliq também foi utilizada como meio contínuo da reação.

Os experimentos foram realizados em uma unidade reacional que consiste num reator convencional encamisado de vidro borossilicato com volume total de $1000 \mathrm{~mL}$, acoplado a um banho termostático que possibilita o controle da temperatura ao longo da reação. A tampa do reator possui cinco entradas para conexões: a haste do agitador, um termopar para o controle da temperatura reacional, a sonda NIR que permite a obtenção de espectros de modo in-situ e online, um condensador, além de um local para a adição dos reagentes. No fundo do reator possui uma saída que permite a retirada de amostras. 


\subsection{Polimerização do Pirrol}

O pirrol necessita ser previamente destilado, para que sejam retiradas as impurezas e evitar a sua oxidação, após este processo purga-se nitrogênio e então é armazenado num refrigerador na temperatura entre $\left(0 \mathrm{C}^{\circ}-5^{\circ} \mathrm{C}\right)$. $\mathrm{O}$ cloreto de ferro hexahidratado são utilizados sem prévia purificação.

Inicia-se a reação com a adição da água miliq no reator, em seguida coloca-se o monômero. Por fim, o cloreto de ferro hexahidratado (agente oxidante) é gotejado na solução, iniciando assim a polimerização do pirrol que possui o tempo reacional de 240 minutos.

Além dos estudos em meio contínuo e estável, também foram realizados testes com pertubação no meio reacional, através da variação do agente oxidante, com o objetivo de monitorar o comportamento reacional ao longo do processo.

A coleta de amostras foi realizada em tempos previamente estabelecidos, onde foram coletadas alíquotas ( \pm 20 gramas) do meio reacional com auxílio de uma seringa dotada de uma tubulação de $\pm 10 \mathrm{~cm}$ de comprimento acoplada a sua ponta. Em seguida a amostra é diluída em uma massa conhecida de água miliq resfriada $\left( \pm 3^{\circ} \mathrm{C}\right)$ e acondicionada em um bécker de $80 \mathrm{~mL}$, esse procedimento faz com que a velocidade da reação seja reduzida, estabilizando o valor da conversão. Segundo relatos na literatura a ideia mais aceita é que a reação é interrompida através de ataque nucleofílico (DIAS et al., 2006).

Com isso, as alíquotas retiradas foram devidamente pesadas em uma balança (Shimadzu modelo AY220) e acondicionadas em papel filtro (Whatman, Cat No 1442 125), sendo realizada uma filtração a vácuo da amostra para a retirada do monômero e dos demais reagentes presentes. Em seguida, o filtrado é levado à estufa de convecção forçada durante um período de 24 horas a uma temperatura de $40^{\circ} \mathrm{C}$. Após esta etapa é obtido o pirrol que é constituído por um pó fino e escuro.

\subsection{Conversão Gravimétrica}

As amostras coletadas ao longo da reação foram úteis para acompanhamento da cinética das reações de polimerização do pirrol, assim como a evolução da conversão. Estes dados foram então empregados na elaboração de modelos de calibração no espectrofotômetro de infravermelho próximo. A conversão gravimétrica $(\mathrm{X})$ do polipirrol é calculada pela Equação 1, que considera a massa do polímero no filtro $(\mathrm{Mpol})$ obtido após a secagem das amostras.

$$
X=\frac{\text { Mpol }}{\left(\frac{\text { Plátex } * \text { MTmon }}{\text { MTreat }}\right)}
$$

Onde:

$\mathrm{X}=$ Conversão gravimétrica;

Mpol = Massa de polímero no filtro;

Plátex = Massa de látex coletado no filtro;

MTmon = Massa total do monômero no reator;

MTreat $=$ Massa total no reator. 


\subsection{Espectrofotômetro de Infravermelho Próximo}

O equipamento utilizado para o monitoramento das reações de polimerização do pirrol foi o espectrofotômetro de infravermelho próximo com Transformada de Fourier (FTNIR) da ABB Bomem modelo FTLA2000-160, que está acoplado a um computador que armazena e processa os dados gerados. A coleta de espectros foi realiza em intervalos regulares ao longo da reação. Um feixe de fibras óticas com aproximadamente 5 metros interliga o equipamento a uma sonda de transflectância, a qual é imersa no meio reacional. A faixa espectral de interesse para fins de calibração situa-se entre $780 \mathrm{~nm}\left(12.820 \mathrm{~cm}^{-1}\right)$ e $2500 \mathrm{~nm}\left(4000 \mathrm{~cm}^{-1}\right)$ e os espectros coletados consistiam no valor médio de 16 varreduras demandando um tempo total de 25 segundos para cada coleta. Para correlacionar as propriedades de interesse com os espectros foi utilizada a técnica de regressão multivariada baseadas em mínimos quadrados parciais (PLS, ou PartialLeastSquares).

\section{RESULTADOS E DISCUSSÕES}

As condições experimentais das corridas de polimerização do pirrol são apresentadas na Tabela 1, os experimentos foram conduzidos a uma temperatura de $20^{\circ} \mathrm{C}$ e sob distintas concentrações molares de agente oxidante. É importante salientar que a relação molar entre agente oxidante e pirrol foi mantida constante. Na Tabela 1 também são exibidas as conversões das corridas experimentais e o teste referente ao aumento gradativo da concentração de cloreto de ferro. Através desses ensaios é possível inferir que a conversão do pirrol decresce de acordo com a concentração inicial dos reagentes, isso ocorre devido à taxa de polimerização decair com a concentração inicial dos reagentes, uma possível explicação para esse fenômeno é que não ocorrem conversões completas para a solução do meio reacional com baixas concentrações de reagentes, mesmo que sejam usados maiores tempos de reação, os trabalhos de LEI et al. (1992) e SONG et al. (2004) corroboram com tais resultados.

Tabela 1 - Condição experimental das corridas do polipirrol a $20^{\circ} \mathrm{C}$.

\begin{tabular}{l|ccccc}
\hline Formulação & $\begin{array}{c}\text { Tempo } \\
\text { Reacional (min) }\end{array}$ & $\begin{array}{c}\text { Conversão } \\
(\%)\end{array}$ & $\begin{array}{c}\text { Temperatura } \\
\left({ }^{\circ} \mathrm{C}\right)\end{array}$ & $\begin{array}{c}{[\mathrm{FeCl} 3} \\
\left.6 \mathrm{H}_{2} \mathrm{O}\right](\mathrm{M})\end{array}$ & $\begin{array}{c}\mathrm{FeCl}_{3} 6 \mathrm{H}_{2} \mathrm{O}: \text { Pirrol } \\
(\mathrm{mol}: \mathrm{mol})\end{array}$ \\
\hline P1 & 240 & 68,4 & 20 & 0,5 & $2,3: 1$ \\
P2 & 240 & 83,5 & 20 & 0,5 & $2,3: 1$ \\
P3 & 240 & 48,8 & 20 & 0,35 & $2,3: 1$ \\
P4 & 240 & 91,5 & 20 & 0,35 & $2,3: 1$ \\
P5 & 240 & 49,7 & 20 & 0,25 & $2,3: 1$ \\
P6 & 240 & 49,4 & 20 & 0,1 & $2,3: 1$ \\
P7 & 240 & 89,8 & 20 & $0,25+0,25$ & $2,3: 1$ \\
\hline
\end{tabular}


Ao longo das corridas foram coletados espectros em intervalos regulares, desde o carregamento do reator até o término da reação, como pode ser observado na Figura 1. No início os valores de absorbância são baixos e ao longo da reação esses valores aumentam devido o consumo dos reagentes e formação do pirrol, nota-se a presença de espectros característicos de partículas em fase sólida, que são retilíneos e sem a presença picos espectrais. Por outro lado, CHICOMA et al. (2011) constataram que a nucleação e o crescimento de partículas produz deslocamentos de linha base dos espectros na região NIR. Ainda na Figura 1, os espectros tornam-se sobrepostos ao final da reação, indicando que tanto a conversão da reação quanto a granulometria polimérica atingiram o estado estacionário.

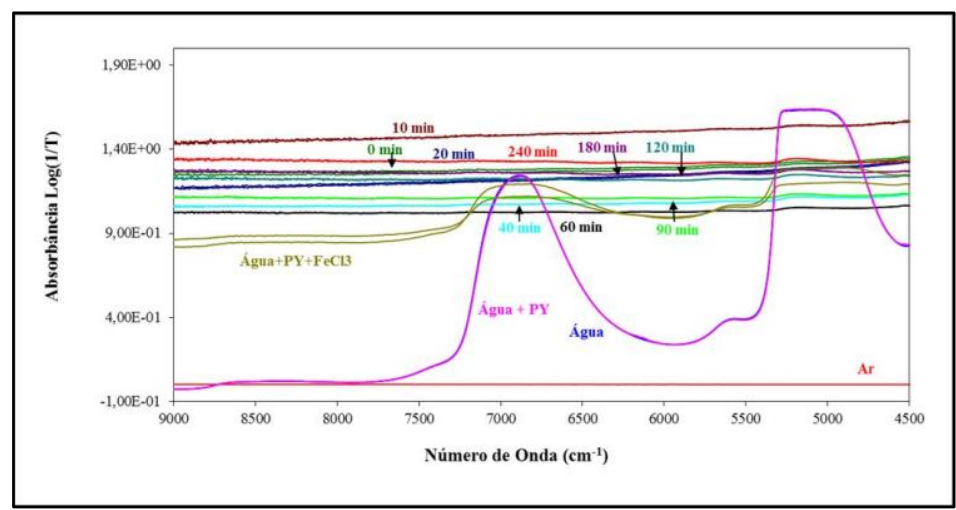

Figura 1 - Espectros NIR coletados ao longo da polimerização do pirrol para o experimento $\mathrm{P} 3$ a $20^{\circ} \mathrm{C}$.

Os dados experimentais selecionados para a calibração do equipamento foram oriundos dos experimentos P1, P2, P3, P4, P5 e P6. O experimento P7 constituiu o banco de dados de validação. De acordo com RICHARD et al. (2011) constata-se que na região de $9000 \mathrm{~cm}^{-1} \mathrm{a}$ absorção de sinais é praticamente inexistente, enquanto que abaixo de $4500 \mathrm{~cm}^{-1}$ aparece uma variação do ruído espectral, no qual essas regiões passam a ser retiradas da figura. A faixa espectral selecionada para este trabalho foi de 4500 a $7500 \mathrm{~cm}^{-1}$, observa-se na Tabela 2 o desempenho do modelo de calibração com correlações intermediárias, através desse estudo foi possível constatar a influencia da opacidade do meio, fenômeno este que influenciou no efeito de espalhamento de luz nessa região.

Tais modelos consideram espectros brutos (dados sem tratamento) ou espectros préprocessados, incluindo tratamentos como $1^{\mathrm{a}}$ e $2^{\mathrm{a}}$ derivadas, que consiste em um dos melhores métodos para a remoção dos efeitos de linha de base. Onde a $1^{a}$ derivada do espectro é a medida da inclinação da curva espectral em cada ponto, sendo um tratamento efetivo para a remoção do deslocamento da linha de base. Um segundo tratamento bastante utilizado na área para inferência de propriedades via espectros se dá pela $2^{a}$ derivada. Esta operação é muito eficaz na remoção do deslocamento da linha de base e da inclinação espectral. O método usado para o cálculo das derivadas é o da diferença simples. Os tratamentos matemáticos foram utilizados no modelo de 
calibração e o número de fatores corresponde ao número de combinações lineares estabelecido pelo algoritmo PLS para o conjunto de dados espectrais (PLSplus IQ, 2004).

Tabela 2 - Resultados de calibração NIR x conversão da polimerização do pirrol oriundos dos experimentos P1, P2, P3, P4, P5 e P6.

\begin{tabular}{l|cccc}
\hline Propriedade & Faixa & Correlação $\left(R^{2}\right)$ & Fatores & Tratamento matemático \\
\hline Conversão (\%) & $0-91,47$ & 0,8110 & 7 & Bruto \\
\hline
\end{tabular}

A validação realizada para o experimento P7 pode ser apreciada na Tabela 3 em conjunto com os erros obtidos para cada modelo e a Figura 2 exibe os resultados de monitoramento da conversão do polipirrol durante o experimento P7. Verifica-se que os modelos são capazes de interpolar razoavelmente os dados experimentais, mostrando predições adequadas para conversão. Acredita-se que o aumento do conjunto de dados de calibração poderia contribuir para a redução das oscilações observadas na predição.

Tabela 3 - Erro padrão de predição (SEP) dos valores experimentais do P7.

\begin{tabular}{lc}
\hline $\begin{array}{r}\text { Tratamento } \\
\text { Matemático }\end{array}$ & $\begin{array}{c}\text { Valores do erro P7 - } \\
\text { Conversão }(\boldsymbol{\%})\end{array}$ \\
\hline SEP - Bruto & 31,19 \\
SEP - 1 $^{\text {a derivada }}$ & $\mathbf{2 6 , 3 7}$ \\
SEP - 2 2 $^{\text {derivada }}$ & 29,13 \\
\hline
\end{tabular}

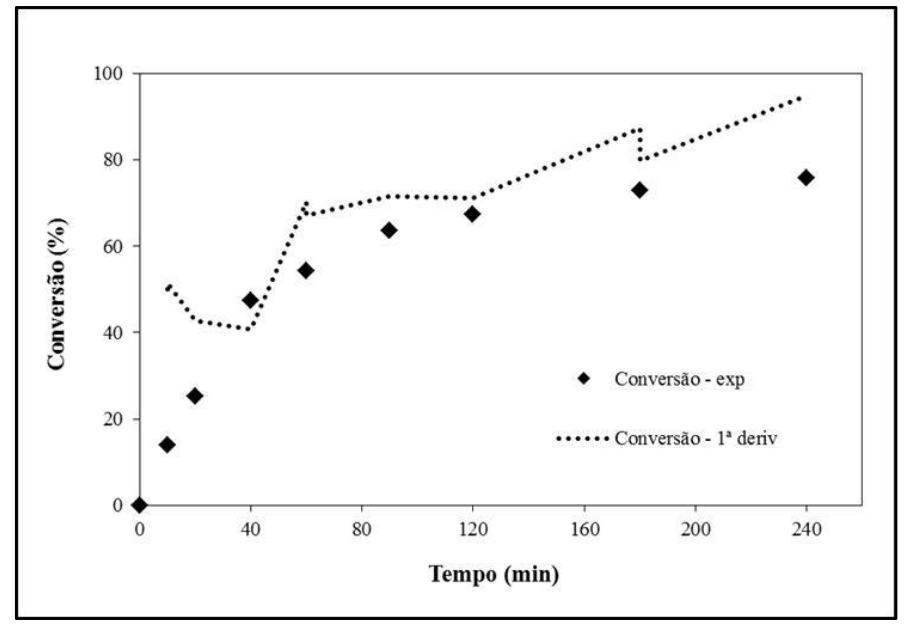

Figura 2 - Monitoramento da polimerização do pirrol via modelos de calibração para o experimento P7. 


\section{9 a 22 de outubro de 2014 \\ Florianópolis/SC}

Cabe ressaltar que o experimento P7 foi submetido ao aumento gradativo de agente oxidante, o que favoreceu a oscilação do nível de opacidade do sistema, observa-se que mesmo sob influências externas o modelo foi capaz de interpolar razoavelmente os dados experimentais.

Ao longo do trabalho é possível identificar que o método de tratamento matemático dos espectros que mais se destaca é o da primeira derivada. Ressalta-se, porém que, em muitos casos, o tratamento matemático dos espectros não produz ganhos significativos na qualidade da calibração/predição.

Apesar dos desafios encontrados ao longo da pesquisa, é possível constatar que o espectrofotômetro é eficaz na identificação da variação da conversão ao longo da reação, observa-se também a necessidade da estruturação de um banco de dados mais complexo e robusto para que possa inferir adequadamente nas propriedades do polipirrol.

\section{CONCLUSÃO}

A espectrofotometria NIR mostrou-se, neste trabalho, uma ferramenta eficaz, mas que necessita ser cada vez mais trabalhada e adaptada para sistemas envolvendo partículas sólidas e meios com bastante opacidade, pois a técnica permite a rápida obtenção de informações relevantes sobre a qualidade do polipirrol produzido durante as reações.

Acredita-se que com um banco de dados mais robusto a metodologia de calibração desenvolvida neste trabalho possa gerar resultados cada vez mais significativos, viabilizando assim a aplicação da técnica NIR em reatores de maior porte.

\section{AGRADECIMENTOS}

Os autores agradecem a Coordenação de Aperfeiçoamento do Nível Superior (CAPES), a Fundação de Apoio a Pesquisa e à Inovação Tecnológica do Estado de Sergipe (FAPITEC/SE) e à Universidade Tiradentes pelo auxílio financeiro e concessão de bolsas de estudos.

\section{REFERÊNCIAS}

CHEN, Y. F.; LIU, J.; YAO, H. J.; MO, D.; DUAN, J.L.; HOU, M. D.; SUN, Y. M.; ZHANG, L.; MAAZ, K. Electrochemical polymerization and characterization of polypyrrole nanowires and nanotubules. Physica B, 405, p. 2461-2465, 2010.

CHICOMA, D.L.; SAYER, C.; GIUDICI, R. In-Line Monitoring of Particle Size during Emulsion Polymerization under Different Operational Conditions using NIR Spectroscopy, Macromol. React. Eng., 5, 150-162, 2011.

DIAS, H.V.R.; MAURO FIANCHINI, M.; RAJAPAKSE, R.M.G. Greener method for high-quality polypyrrole. Polymer, 47, p. 7349-7354, 2006.

EOFINGER, S.; VAN OOJI, W.J.; RIDGWAY, T.H. DC plasma-polymerization of pyrrole: 
Comparison of films formed on anode and cathode. Journal of Applied Polymer Science, 61, p. 15031514,1998 .

FONSECA, G. E.; DUBÉ, M. A.; PENLIDIS, A. A critical overview of sensors for monitoring polymerizations. Macromolecular Reaction Engineering, 3, 327-373, 2009.

GOSPODINOVA, N.; TERLEMEZYAN, L. Conducting polymers prepared by oxidative polymerization: polyaniline, Progress in Polymers science, 23, p. 1443-1484, 1998.

LASCELLES, S.F.; MCCARTHY, G.P.; BUTTERWORTH, M.D.; ARMES, S.P. Effect of synthesis parameters on the particle size, composition and colloid stability of polypyrrole-silica nanocomposite particles. Colloid Polymer Science, 276, p. 893-902, 1998.

LEI, J.; CAI, Z.; MARTIN, C. R. Effect of reagent concentrations used to synthesize polypyrrole on the chemical characteristics and optical and electronic properties of the resulting polymer. Synthetic Metals, 46, p. 53-69, 1992.

PLSplus IQ, Thermo electron corporation, 2004.

PRON, A.; RONNOU, P. Processible conjugated polymers: from organic semiconductors to organic metals and superconductors, Progress in Polymers Science, 27, p. 135-190, 2002.

RICHARD, R.; LI, Y.; DUBREUIL, B.; THIEBAUD-ROUX, S.; PRAT, L. On-line monitoring of the transesterification reaction between triglycerides and ethanol using near infrared spectroscopy combined with gas chromatography, Bioresource Technology, 102, p. 6702-6709, 2011.

SANTOS, A.F.; SILVA, F.M.; LENZI, M.K.; PINTO, J.C. Monitoring and control of polymerization reactors using NIRS, Polymer-Plastics Technology and Engineering, 44, p. 1-61, 2005.

SKOTHEIM, T.A.; ELSENBAUMER, R.L.; REYNOLDS, J.R. Handbook of conducting polymers. 3nd ed. NY: Marcel Dekker, 2007.

SONG, M.K; KIM, Y.T.; KIM, B.S.; KIMC, J.; CHAR, K., RHEE, H.W. Synthesis and characterization of soluble polypyrrole doped with alkylbenzenesulfonic acids. Synthetic Metals, 141, p. 315-319, 2004.

SYED, A.A.; DINESON M.K. Polyaniline- A novel polymeric material- review, Talanta, 38, p. 815837, 1991.

VERNITSKAYA, T.V.; EFIMOV, O.N. Polypyrrole: a conducting polymer; its synthesis, properties and applications, Russian Chemical Reviews, 66, p. 443 - 457, 1997. 